Case Report

\title{
Enhanced Mental Health with Virtual Reality Mental Hygiene by a Veteran Suffering from PTSD
}

\author{
Bo Søndergaard Jensen $\left(\mathbb{D},{ }^{1}\right.$ Niels Andersen, ${ }^{2}$ Jes Petersen, ${ }^{3}$ and Lene Nyboe $\mathbb{D}^{1}$ \\ ${ }^{1}$ Clinic for PTSD and Anxiety, Aarhus University Hospital Skejby, Denmark \\ ${ }^{2}$ DTU Space, Denmark \\ ${ }^{3}$ PrimeView Film Production, Denmark \\ Correspondence should be addressed to Bo Søndergaard Jensen; bojensen@rm.dk
}

Received 9 February 2021; Revised 6 June 2021; Accepted 21 June 2021; Published 5 July 2021

Academic Editor: Lut Tamam

Copyright (c) 2021 Bo Søndergaard Jensen et al. This is an open access article distributed under the Creative Commons Attribution License, which permits unrestricted use, distribution, and reproduction in any medium, provided the original work is properly cited.

\begin{abstract}
This paper describes the application and feasibility of the use of Virtual Reality Mental Hygiene (VRMH) as a mean to reduce anxiety and stress in a Danish veteran suffering from posttraumatic stress disorder (PTSD) and enduring personality change after a catastrophic experience. The results from this case study provide preliminary evidence that VRMH can be used as a mean to reduce arousal in patients with severe PTSD.
\end{abstract}

\section{Introduction}

Posttraumatic stress disorder (PTSD) is a mental disorder that can occur after severe or prolonged mental trauma of an exceptionally threatening or catastrophic nature such as serious accidents, war, natural disasters, or rape. PTSD is characterized by reexperiencing the traumatic event in the form of invasive memories, flashbacks, and nightmares. The patient experiences severe anxiety when exposed to circumstances reminiscent of the traumatic event and avoidance of anything reminiscent of the trauma, both physically and mentally, is common. There are persistent symptoms of mental hypersensitivity and alertness characterized by difficulty falling asleep, irritability, difficulty concentrating, hypervigilance, and tendency to risk-seeking behaviour (e.g., driving recklessly, taking drugs, and having unprotected sex).

PTSD is often a relatively chronic disorder, and about $1 \%$ of the population suffers from PTSD at any given time. Among soldiers who have been at war, as well as victims after disasters, the incidence of PTSD is about $10 \%$ and among victims of rape about $30 \%[1,2]$.

Exposure therapy as a treatment of PTSD has received significant support from many randomized controlled trials as well as meta-analyses. A Cochrane meta-analysis con- cluded that individual trauma-focused cognitive behavioural therapy (TFCBT), EMDR, stress management, and group TFCBT are effective in treating PTSD, whereas other nontrauma-focused psychological treatments do not reduce PTSD equally significantly [3].

Prolonged exposure therapy (PE), which is an individual TFCBT, has been shown to be effective in treating PTSD from various types of traumatic events including combat actions [4-6]. APA's clinical guidelines from 2017 as well as NICE guidelines from 2018 strongly recommend the use of PE in the treatment of PTSD $[7,8]$.

The dropout rate for treatment with PE is between 13\% and $38 \%$ [9], and $20-50 \%$ of those who complete the treatment maintain their PTSD diagnosis despite significant symptom reduction [10-13]. So, even though the treatment of PTSD with PE has proved promising, there is still a need for further improvements in treating patients with PTSD. Therefore, there is a basis for investigating potential methods in addition to the existing treatment. One possible intervention could be Virtual Reality Mental Hygiene (VRMH) as a mean to reduce stress and anxiety.

In this case study, the project hypotheses were that VRMH could activate the soothing system and the parasympathetic nervous system in the patient, and this activation 
could have a positive impact on the general mental health and overall well-being.

The intervention manual was developed within the theoretical framework of compassion-focused therapy [14, 15]. The modern human brain is a product of a long evolution, in which different evolutionary conditions have formed the brain, with the essential purpose of survival. Different parts of the brain reflect different motivations, abilities, and interests, and this affects our experiences and the way we live our lives in different ways.

The less evolved part of the human brain, the reptilian brain, is concerned with territory, food, reproduction, and survival. The more evolved mammalian brain is concerned with living in groups, hierarchy, status, and caring. The most evolved part of the human brain is concerned with extended caregiving, attachment, and thinking.

These different parts of the brain are linked to our three major emotional regulation systems, the threat system, the drive system, and the soothing system. Each of the emotional regulation systems is associated with distinct states of feeling, motivations, behaviour, neuroanatomy, and neurochemistry. Healthy functioning requires adaptive use of all three systems in appropriate measures. Dysfunction can occur due to limited flexibility or overuse of one system to the detriment of others [16].

When the threat system is dominant, our mind is focused on seeking protection from dangers motivated for survival. Our emotions are about fear and anxiety, and our physiology is highly aroused with a behaviour dominated by fight or flight.

When the drive system is dominant, our mind is attuned towards wanting, seeking, aspiring, or striving. Our emotions are positive and motivated, and our physiology is aroused with a behaviour dominated by focus on specific goals.

When the soothing system is dominant, our mind is attended to giving and receiving care, affection, and nurturance. Our emotions are about safeness, and our physiology is calm with a behaviour dominated by looking-after and soothing.

The three emotional regulation systems are connected to the human autonomic nervous system. The threat system and drive system are mainly connected to the sympathetic nervous system, and the soothing system is mainly connected to the parasympathetic nervous system $[16,17]$.

The sympathetic nervous system is responsible for the fight or flight response. In the first moments after a stressor occurs, the hypothalamic pituitary adrenal axis (HPA axis) is stimulated. The HPA axis is responsible for the neuroendocrine adaptation component of the stress response. The response is characterized by secretion of cortisol, adrenaline, and noradrenaline. It increases pulse, breathing, and cardiovascular efficiency and dilates the bronchioles and pupils. It also divides fat into fatty acid and glycerol, breakdowns glycogen stored in liver to glucose, causes outflow of blood from the limbs to muscles, heart, and brain, and stops digestion. All this is to prepare the body for a fight or flight response.

At a certain blood concentration of cortisol, adrenaline, and noradrenalin, this protection response is achieved, and the there is a negative feedback to the HPA axis and systemic homeostasis returns. The parasympathetic nervous system is responsible for the body's rest and digestion response in which the body is relaxed, resting, or feeding. It basically undoes the work of sympathetic division after a stressful situation. The parasympathetic nervous system decreases respiration and heart rate and increases digestion.

Our sympathetic nervous system is designed to help us to survive in life-threatening emergencies, but if we spend too long in this heightened state of sympathetic activation, this can have negative consequences on our health. We will become exhausted, unsettled, experience cognitive decline, and experience poor sleep, and the immune system will be compromised. In the long run, this repeated exposure to stressors and activation of the sympathetic nervous system will also cause habituation of the organism to the stressor with repeated and sustained HPA activation. Therefore, it is important to support a healthy balance between sympathetic and parasympathetic activation.

One way to achieve this is through the activation of the soothing system and thereby activation of the parasympathetic nervous system. Our soothing system can be activated in various ways, e.g., through positive interaction with other humans, slow-breathing, mindfulness, yoga, body awareness therapy, positive self-talk/letter writing, and visualization exercises $[16,17]$.

\section{Case Presentation}

2.1. Participant. The patient included was a 45 -year-old male Danish war veteran who had been in treatment for chronic PTSD and depression in the psychiatric outpatient clinic for PTSD and Anxiety at Aarhus University Hospital Skejby, Denmark.

Prior to his deployment to Bosnia in 1995 for a period of 6 months as part of the Danish UN mission, the patient was mentally inconspicuous. He describes the childhood home as safe, a place where he had received care and attention, and there were no problems with alcohol or violence in the family. He has generally had good relationships with other people and had few close friends. The patient had no mental health difficulties and had no mood swings or anxiety.

After the deployment, the patient was unemployed for about 7 months, before he got a new contact with the Armed Forces, where he served for a period of 4 years. After that, the patient had several shorter employments, from 4 months to 2 years, as an unskilled worker. Among other things, he was a factory worker, guard, doorman, slaughterhouse worker, and most recently a truck driver. He has been unemployed since 2012, and in 2019, he granted an early retirement benefit due to his mental health condition. After his deployment, he subsequently had an increase in mental health difficulties and a changed behaviour with mood swings, anxiety, and a pronounced tendency to distrust of other people. His mental problems had severe social consequences, partly in the form of a decline in his social network, periodic abuse of alcohol and drugs, and several times of disulfiram treatment of alcoholism.

Before the treatment at the Clinic for PTSD and Anxiety, the patient's PTSD had been treated with several different 
psychotropic medications, mainly SSRI and SNRI drugs. The patient sought treatment for the first time in 2001.

At intake to the clinic, the patient was unemployed and living alone. He had divorced his wife several years before. He had two children from this marriage but had little contact with them at the beginning of the treatment.

Initially in treatment, the patient described several psychotraumatizing incidents from his deployment to Bosnia. These included war-related experiences such as shootings, explosions, fires, bombing, and being taken hostage and used as a bomb shield for several days.

In relation here to, the patient had repeated stressful intrusive memories of these experiences, e.g., nightmares dealing with incidents from his deployment. He did not describe any episodes of flashback or loss of reality. He became intensely emotionally upset when he was reminded of the traumatic events describing different directed emotions such as anxiety, anger, and sadness. The patient reported suffering from daily feelings of anxiety. He experienced discomfort when exposed to circumstances reminiscent of the trauma. The anxiety worsened when he was in larger gatherings of people and had panic anxiety accompanied by autonomic symptoms in the form of palpitations, chest tightness, and headaches. Together, the above symptoms led to a pronounced tendency to isolation. Thus, the symptom picture met the ICD-10 criteria for PTSD.

Firstly, the PTSD symptoms were treated with prolonged exposure therapy (PE) with a significant reduction of symptoms, especially the invasive symptoms. Although there was a significant reduction of symptom level, he still met the diagnostic criteria for PTSD. Specifically, his symptoms of hypervigilance were not changed. Further, he experienced a persistent change in personality.

After his deployment, he became significantly more distrustful and hostile to the outside world, had severe difficulties being with other people, and had chronically increased tension, as if he was always facing a threatening situation, even though he objectively knows well that the situation is not dangerous or that other people will not hurt him. He had a persistent feeling of emptiness and hopelessness and feelings of being alienated. Thus, there were clear signs of a personality change accordingly to WHO's ICD-10 diagnosis F62.0 enduring personality change after catastrophic experience.

The patient agreed to participate in this pilot study examining whether Virtual Reality Mental Hygiene (VRMH) could be a possible intervention to reduce stress and anxiety for patients with PTSD. He was reassured about confidentiality by emphasizing that information would not be made available to a third party without his permission, and that results would only be used for publication in professional journals. The patient gave informed consent for participation in the study, in accordance with the Helsinki Declaration.

2.2. Intervention. In this study, the VRMH stimulation comprised a naturalistic 10-minute virtual reality film of the beach by the west coast of Denmark with a 360-degree view. This was chosen in agreement with the patient as he had previously perceived this as a calming and soothing environment. The video was accompanied by the sound of waves from the ocean with the purpose of giving a more naturalistic experience, in all designed to activate the soothing system and the parasympathetic nervous system in the patient.

The VR headset used in the study was a pair of PIMAX $4 \mathrm{~K}$ with $1000 \mathrm{~Hz}$ dual gyroscope with $18 \mathrm{~ms}$ MTP (motion to photons) and a 360-degree FOV (field of view). The PC had an Intel Core i5-7500 CPU with $3.40 \mathrm{GHz}$ and $8.00 \mathrm{~GB}$ RAM. The graphics card was a GeForce GTX 10603 GB.

During two months of study, the patient was instructed to use VRMH every afternoon. Further, the patient was instructed to be aware of any side effects when using the VR glasses, e.g., vertigo. During the two months, the patient had weekly sessions with his psychologist giving the possibility to address side effects. Apart from vertigo and in rare occasions nausea, there are no known side effects of using VR. For this patient, VR was seen to be an easy and applicable treatment for addressing his persistent feelings of hypervigilance that had not improved sufficiently during treatment.

Before the study, the patient's PTSD and comorbid depression had been treated with prolonged exposure therapy and $200 \mathrm{mg}$ of Sertraline with a significant reduction of PTSD symptoms. For a period of two months before and during the VRHM study, there were no changes in pharmacotherapy.

2.3. Assessment. Psychiatric status was assessed using the Mini-International Neuropsychiatric Interview (M.I.N.I.), which is a short structured diagnostic interview developed by psychiatrists and clinicians in the United States and Europe for DSM-IV and WHO's ICD-10 psychiatric disorders. The M.I.N.I is screening for 17 most common disorders in mental health according to DSM-IV and ICD-10.

The disorders investigated are the most important to identify in clinical and research settings.

For each disorder, one or two screening questions rule out the diagnosis when answered negatively.

It was designed to meet the need for a short but accurately structured psychiatric interview for multicenter clinical trials and epidemiology studies and has an administration time of approximately 15 minutes [18].

For measuring the effect before and after the use of VRMH, a self-report questionnaire was developed for assessing feelings of anxiety, stress, vigilance, anger, and sadness. On one side of the self-report questionnaire, the patient was instructed to mark on a $10 \mathrm{~cm}$ long visual analog scale (VAS) from zero to ten, where zero marked not at all and ten marked very much, how much the item bothered him, before the use of VRMH, and on the other side with an identical self-questionnaire, how much the item bothered him after the use of VRMH, without looking at the previous rating.

Differences between the assessment before and after VRMH were statistically analyzed using paired $t$-test, with a significance level of 0.05 . All analyses were performed in SPSS ver.20.

\section{Results}

In all, the patient performed 40 daily assessments before and after use of VRMH, and the statistical analyses are based on these. Besides feelings of "stress," all items showed statistically 
TABLE 1: Mean differences between before and after use of VRMH.

\begin{tabular}{lcccc}
\hline Item & Before $($ mean $(\mathrm{SD}))$ & After $($ mean $(\mathrm{SD}))$ & Mean difference $(\mathrm{SD})(95 \% \mathrm{CI})$ & $p$ value \\
\hline Anxiety & $3.54(0.47)$ & $3.37(0.44)$ & $0.17(.43)(0.03 ; 0.31)$ & .02 \\
Stress & $3.57(0.48)$ & $3.50(0.49)$ & $0.06(0.37)(-0.05 ; 0.18)$ & .26 \\
Vigility & $4.44(0.63)$ & $4.23(0.68)$ & $0.22(0.48)(0.07 ; 0.38)$ & .006 \\
Anger & $3.72(0.73)$ & $3.45(0.74)$ & $0.27(0.60)(0.08 ; 0.46)$ & .007 \\
Sadness & $3.52(0.82)$ & $3.23(0.66)$ & $0.29(0.65)(0.08 ; 0.49)$ & .009 \\
\hline
\end{tabular}

significant reductions from before to after use of VRMH (Table 1). The feeling of "stress" seemed to worsen after use of VHMR; however, this was not statistically significant.

\section{Discussion}

In this single case study, the use of VRMH significantly reduced feelings of anxiety, vigilance, anger, and sadness in a Danish war veteran with an ICD-10 diagnosis of PTSD (F43.1) and an ICD-10 diagnosis of enduring personality change after catastrophic experience (F62.0). The case study therefore provided preliminary evidence that VRMH can be useful in reducing symptoms of arousal and anxiety in a Danish veteran with chronic PTSD. However, the feeling of stress seemed to worsen after use of VRMH. A possible explanation for this could be that the item "feelings of stress" is not so clearly defined as other feelings being assessed. It could also be due to the fact that sitting quietly when wearing the VR equipment presents a genuine stress factor for this particular patient. Previously, VR has mainly been applied to patients with PTSD as an exposure to traumatic events, e.g., war [19]. In accordance with our results, a recent study of VR of nature videos improved feelings of anxiety and stress in patients with severe psychiatric disorders [20]. However, it cannot be ruled out that a large proportion of the intervention effect can be attributed to nonspecific factors and not to the specific method. Nonspecific factors such as the therapeutic alliance and perceived social support could have influenced the outcome more than the specific intervention.

Another limitation of the study is that there was no longtime follow-up with measurements of more psychiatric symptoms as, e.g., PTSD and depression.

The use of biological measurement during the use of VRMH could also strengthen the validity of the collected data. These could be measurements of galvanic skin response (GSR) or heart rate variability (HRV).

GSR is an autonomic respond in the sweat glands of the skin, controlled by the sympathetic nervous system (SNS), and the skin conductance is an indication of physical and psychological arousal. If the SNS is activated, the sweat gland activity is also activated, and higher arousal in the SNS also increases sweat gland activity and thereby increases the skin conductance; hence, GSR can be a measurement for emotional responses, and a clinical interview or questionnaire could verify what kind of emotion had caused the response [21].

HRV could be another biological maker for the effectiveness of VRMH stimulation to promote general mental health. $\mathrm{HRV}$ is the variation in the time interval between consecutive heartbeats, and HRV represents the ability of the heart to respond to different psychological and physiological stimuli in the environment. Low HRV is associated with impaired regulation of the ANS, and a reduced ability to cope with internal and external stressors and high-resting HRV has been associated with physical and mental health.

Neuroimaging studies suggested that HRV may be linked to cortical regions as the ventromedial prefrontal cortex (vmPFC) and the dorsolateral prefrontal cortex (dlPFC). The vmPFC is involved in stressful situation appraisal, and several studies have provided support for the important role that the vmPFC plays as a moderator and inhibitor of the amygdala.

The dual inhibition model posits that the vmPFC and the dlPFC, along with their associated inhibitory pathways, must interact for adequate inhibitory control of the amygdala and emotional regulation in PTSD $[22,23]$.

The reduction in this VRMH study of feelings of anxiety, vigilance, anger, and sadness could be attributed to a positive stabilization of the HRV, but this is still unclear and needs further study during the application of the VRMH.

Based on the above, VRMH could be an intervention for further investigation as mean to enhance general mental health by patients with chronic PTSD.

Clearly, results from a case study have limited generalization, and there is a need for further research into the use of VRMH. With the addition of biological measurements as GSR and HRV, it could be confirmed whether or not the perceived reduction in anxiety is a result of a decreased activation of the SNS. It would also be of interest if the VRMH has a lasting impact on the HRV, so follow-up studies with measurements of the HRV could strengthen the quality of future studies.

In addition, further research conducted in a general psychiatric clinic with outpatient care could strengthen the general evaluation regarding the utility and capacity of this intervention to both reduce psychopathology and promote general mental health under controlled conditions (efficacy) and to be successful when implemented in routine treatment settings (effectiveness).

\section{Data Availability}

Data is available on request through the first author.

\section{Conflicts of Interest}

The authors declare that they have no conflicts of interest. 


\section{References}

[1] J. Sareen, "Posttraumatic stress disorder in adults: impact, comorbidity, risk factors, and treatment," Canadian Journal of Psychiatry, vol. 59, no. 9, pp. 460-467, 2014.

[2] L. K. Richardson, B. C. Frueh, and R. Acierno, "Prevalence estimates of combat related PTSD: a critical review," The Australian and New Zealand Journal of Psychiatry, vol. 44, no. 1, pp. 4-19, 2010.

[3] J. Bisson and M. Andrew, "Psychological treatment of posttraumatic stress disorder (PTSD)," Cochrane Database of Systematic Reviews, 2007.

[4] B. O. Rothbaum, E. A. Meadoes, P. Resick, and D. W. Foy, "Effective treatments for PTSD: Practice guidelines from the International Society for Traumatic Stress Studies," in Guilford Press, New York, 2000.

[5] M. Yoder, P. T. Tuerk, P. Price et al., "Prolonged exposure therapy for combat-related posttraumatic stress disorder: comparing outcome for veterans of different wars," Psychological Services, vol. 9, no. 1, p. 16, 2012.

[6] Institute of Medicine (IOM), Treatment of Posttraumatic Stress Disorder: An Assessment of the Evidence, The National Academies Press, Washington, DC, 2008.

[7] American Psychological Association, Clinical Practice Guideline for the Treatment of Posttraumatic Stress Disorder (PTSD) in Adults, American Psychological Association, 2017.

[8] National Institute for Health and Care Excellence, Post-traumatic Stress Disorder NG116, 2018.

[9] M. M. Steenkamp and B. T. Litz, "Psychotherapy for militaryrelated posttraumatic stress disorder: Review of the evidence," Clinical Psychology Review, vol. 33, no. 1, pp. 45-53, 2013.

[10] S. A. Rauch, E. Defever, T. Favorite et al., "Prolonged exposure for PTSD in a Veterans health administration PTSD clinic," Journal of Traumatic Stress, vol. 22, no. 1, pp. 60-64, 2009.

[11] P. W. Tuerk, M. Yoder, A. Graubaugh, H. Myrick, M. Hamner, and R. Acierno, "Prolonged exposure therapy for combatrelated posttraumatic stress disorder: an examination of treatment effectiveness for veterans of the wars in Afghanistan and Iraq," Journal of anxiety disorders, vol. 25, no. 3, pp. 397-403, 2011.

[12] E. B. Foa, J. R. Davidson, and A. Frances, "The expert consensus series: treatment of posttraumatic stress disorder," The Journal of Clinical Psychiatry, vol. 60, pp. 4-76, 1999.

[13] J. A. Cigrang, S. A. Rauch, L. L. Avila et al., "Treatment of active-duty military with PTSD in primary care: early findings," Psychological Services, vol. 8, no. 2, pp. 104-113, 2011.

[14] P. Gilbert, "Introducing compassion focused therapy," Advances in Psychiatric Treatment, vol. 15, no. 3, pp. 199208, 2009.

[15] P. Gilbert, "Developing a Compassion-Focused Approach in Cognitive Behavioral Therapy," Cognitive behavior therapy: A guide for the practicing clinician, vol. 2, pp. 205-220, 2009.

[16] P. Gilbert, Compassion Focused Therapy: Distinctive Features, Routledge, 2010.

[17] P. Gilbert, "An introduction to compassion focused therapy in cognitive behavior therapy," International Journal of Cognitive Therapy, vol. 3, no. 2, pp. 97-112, 2010.

[18] Y. Lecrubier, D. Sheehan, E. Weiller et al., "The Mini International Neuropsychiatric Interview (M.I.N.I.): a short diagnostic structured interview: reliability and validity according to the CIDI," European Psychiatry, vol. 12, no. 5, pp. 224-231, 1997.

[19] D. Boeldt, E. McMahon, M. McFaul, and W. Greenleaf, "Using virtual reality exposure therapy to enhance treatment of anxiety disorders: identifying areas of clinical adoption and potential obstacles," Frontiers in Psychiatry, vol. 10, p. 773, 2019.

[20] W. Veling, B. Lestestuiver, M. Jongma, H. R. Hoenders, and C. van Driel, "Virtual reality relaxation for patients with a psychiatric disorder:crossover randomized controlled trial," Journal of Medical Internet Research, vol. 23, no. 1, 2021.

[21] S. Mahima, K. Sudhanshu, and S. Mohit, "A brief introduction and review on galvanic skin response," International Journal of Medical Research Professionals, vol. 2, no. 6, pp. 13-17, 2016.

[22] H. G. Kim, E. J. Cheon, D. S. Bai, L. Young, and B. H. Koo, "Stress and heart rate variability: a meta-analysis and review of the literature," Psychiatry investigation, vol. 15, no. 3, pp. 235-245, 2018.

[23] D. Andrewes and L. Jerkins, "The role of the amygdala and the ventromedial prefrontal cortex in emotional regulation: implications for post-traumatic stress disorder," Neuropsychology review, vol. 29, no. 2, pp. 220-243, 2019. 